\title{
Alcohol screening a brief intervention: a self-paced program for nurses
}

\author{
Deborah Finnell ${ }^{1 *}$, Ann M Mitchell ${ }^{2}$, Christine L Savage ${ }^{1}$, Irene Kane ${ }^{2}$, Robert Kearns ${ }^{1}$, Nathan Poole', \\ Hilda Rizzo-Busack', Scott Coulson ${ }^{2}$ \\ From INEBRIA 12th Congress, \\ Atlanda, GA, USA. 24-25 September 2015
}

\section{Background}

Nurses are in key positions to plan and implement alcohol screening and brief intervention (aSBI). To simultaneously advance aSBI of nurses in various roles, we developed a self-paced program for Clinical Nurse Leaders, Nurse Informaticists, Nurse Administrators, and Registered Nurses including Advanced Practice Nurses. The content for the program is based on the Centers for Disease Control \& Prevention (CDC) 2014 Planning and Implementation Screening and Brief Intervention for Risky Alcohol Use: A Step-by-Step Guide for Primary Care Practices. The objective is to present the results of a cooperative agreement between the Centers for Disease Control \& Prevention, Johns Hopkins School of Nursing, and the University of Pittsburgh School of Nursing. The framework used to design the aSBI Program and key aspects of the program will be demonstrated and discussed.

\section{Material and methods}

The aSBI Program flow, depicted in the CDC guide defined the modules for learning: patient population, assess alcohol consumption, negative screen (and subsequent conversation), positive screen (and assessment of harm and dependence), brief intervention and referral to treatment. The modules were developed using Articulate Storyline, the premier rapid e-learning development platform, and hosted on a learning management system. Special focus was given to the assessment portion of the e-learning modules to ensure learners are provided with authentic assessments that accurately measure their mastery of real-world skills needed to be successful when applying the module educational materials.

\section{Results}

When finalized, the self-paced aSBI Program, funded by the American Association of Colleges of Nursing through the $\mathrm{CDC}$, will be widely disseminated to the nursing community across the U.S.

\section{Conclusions}

This easily accessible on-line educational program will bring evidence-based alcohol screening and intervention to current and future nurses.

\section{Acknowledgements \\ This educational program was supported by a Cooperative Agreement from The Centers for Disease Control and Prevention (CDC RFA OE12-1201 AACN: 1 U36 OE00003-01). Its contents are solely the responsibility of the authors and do not necessarily represent the official views of the Centers for Disease Control and Prevention or the Department of Health and Human Services. Funding support was provided through the American Association of Colleges of Nursing/Centers for Disease Control and Prevention to Johns Hopkins School of Nursing (01-117-WIPFA 14-JU) and the University of Pittsburgh School of Nursing (02-117WIPFA 14-UPITT).}

\section{Authors' details}

'Johns Hopkins School of Nursing, Baltimore, MD, USA. ²University of Pittsburgh School of Nursing, Pittsburgh, PA, USA.

Published: 24 September 2015

\section{Reference}

1. Centers for Disease Control and Prevention: Planning and implementing screening and brief intervention for risky alcohol use: A step-by-step guide for primary care practices Atlanta, GA: Center for Disease Control and Prevention, National Center on Birth Defects and Developmental Disabilities; 2014

doi:10.1186/1940-0640-10-S2-O18

Cite this article as: Finnell et al:: Alcohol screening a brief intervention: a self-paced program for nurses. Addiction Science \& Clinical Practice 2015 10(Suppl 2):018.

\footnotetext{
* Correspondence: dfinnel1@jhu.edu

1Johns Hopkins School of Nursing, Baltimore, MD, USA

Full list of author information is available at the end of the article
} 